\title{
Novel Antenna Concept for Compact Millimeter-Wave Automotive Radar Sensors
}

\author{
Stefan Beer, Grzegorz Adamiuk, Student Member, IEEE, and Thomas Zwick, Senior Member, IEEE
}

\begin{abstract}
An antenna concept for compact millimeter-wave radar sensors that desire a fan-beam radiation pattern is proposed. It combines an end-fire antenna on a ceramic substrate with a cylindrical parabolic reflector that can be integrated in the sensor's metal housing. The concept allows a direct interconnection from the active components to the antenna in a flip-chip attachment. It is validated with a $77-\mathrm{GHz}$ Yagi-Uda antenna on a 127- $\mu \mathrm{m}$ thick Alumina substrate by measurements with a probe-based measurement setup. The measured return loss, gain, and radiation patterns of the antenna without and with the reflector are given.
\end{abstract}

Index Terms-End-fire antennas, millimeter-wave antennas, millimeter-wave radar, radar antennas, reflector antennas.

\section{INTRODUCTION}

$\mathbf{T}$ HE use of radar sensors for driver assistance systems has become widespread in the last years [1]. By now, the focus in the development of automotive radar sensors mainly lies in the allocated frequency bands at $76-77 \mathrm{GHz}$ for long-range radar (LRR) [2] and $77-81 \mathrm{GHz}$ for short-range radar (SRR) [3]. To achieve a high angular resolution, digital beamforming (DBF) concepts are expected to be used in future systems [1], [4], [5]. These require a fan-beam radiation pattern with a narrow beam in the elevation plane and a wider beam in the azimuth plane.

While at the moment millimeter-wave radars are only used in high-class and in some middle-class vehicles, the aim is to produce cost-efficient sensors that could even be used in compact cars. To achieve this goal, the size and the cost of these sensors have to be further reduced while maintaining high performance. The antenna concept used usually plays an important role regarding both cost and size of the sensor. If columns of patches are used as transmit and receive antennas [1], a large substrate area has to be used to realize the effective antenna area needed for the narrow beam in the elevation plane. To achieve a cheaper sensor, it would be desirable to minimize the substrate area needed for the antenna and to use only one substrate for the antenna and the other RF circuitry. The use of a cylindrical lens for beam-shaping and to reduce the size of the patch columns has been proposed [6], but this would lead to the unwanted side-effect of an increased installation depth.

Manuscript received May 12, 2009; revised June 10, 2009. First published July 07, 2009; current version published July 28, 2009.

The authors are with the Institut fuer Hochfrequenztechnik und Elektronik (IHE), Universitaet Karlsruhe (TH), 76131 Karlsruhe, Germany (e-mail: stefan. beer@ihe.uka.de; grzegorz.adamiuk@ihe.uka.de; thomas.zwick@ihe.uka.de).

Color versions of one or more of the figures in this letter are available online at http://ieeexplore.ieee.org.

Digital Object Identifier 10.1109/LAWP.2009.2026917

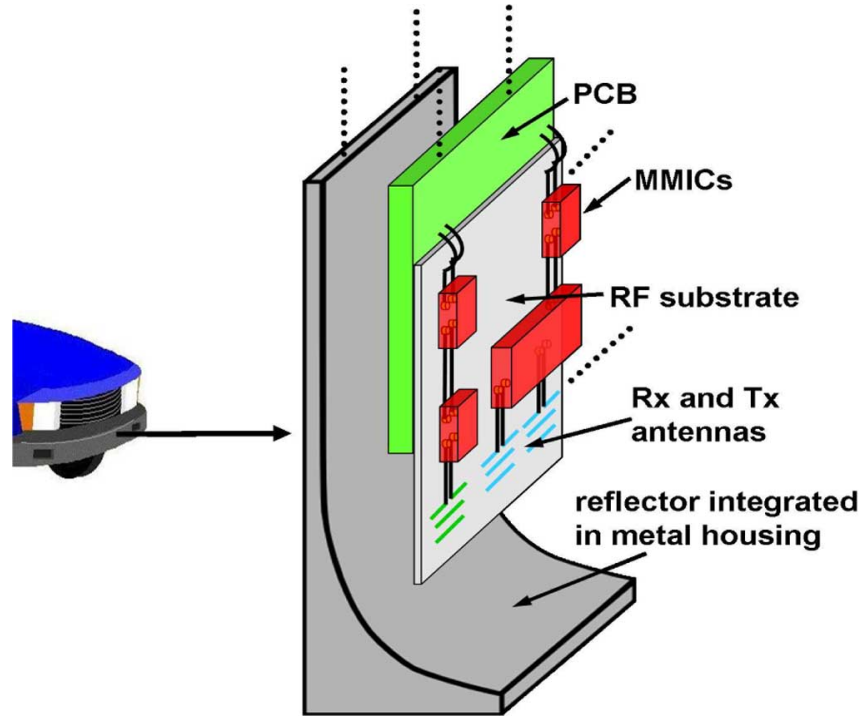

Fig. 1. Radar antenna concept with end-fire antennas and a cylindrical parabolic reflector.

As a lot of progress has been achieved in the realization of highly integrated RF circuits [7], and even in the integration of digital and baseband circuitry into small modules, it can be expected that these devices will further be miniaturized. In that case, an antenna concept that needs a smaller substrate area and that allows a direct connection from the active components to the antenna while maintaining a small installation depth is desired.

\section{PRoposed Antenna Concept}

\section{A. Overall Layout}

To achieve the desired properties, we propose an antenna concept that combines an end-fire antenna and a cylindrical parabolic reflector, as shown in Fig. 1. The end-fire antenna is realized on a RF substrate, and its main lobe points to the side of the sensor's metal housing, in which a cylindrical parabolic reflector is directly integrated. It reflects the radiation to the desired direction perpendicular to the circuit board and, at the same time, focuses the beam in the elevation plane.

The antenna substrate is used as the RF board at the same time, and all monolithic microwave integrated circuits (MMICs) can be attached to the substrate in a flip-chip interconnect. This way, a short and low-loss path for the millimeter-wave signal is guaranteed. The RF substrate is directly attached to the printed circuit board (PCB), and the low frequency and dc interconnects can be realized by bond wires. For the different receive and transmit antennas, either a single antenna or an antenna array 


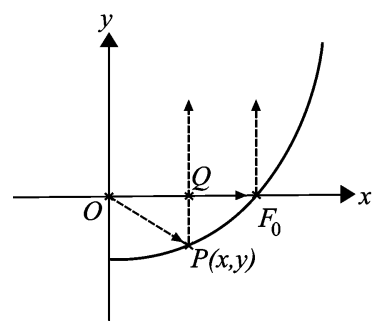

Fig. 2. Cross section of the cylindrical parabolic reflector.

can be used depending on the desired azimuth beamwidth. Generally, the PCB is populated with circuit elements on both sides, so it makes sense to position it at about half the height of the reflector. This configuration can be achieved by choosing the adequate focal distance for the reflector.

The proposed antenna concept could also be realized in a different circuit layout. A possibility is a configuration with a multilayer substrate like LTCC or LCP, in which the MMICs are integrated in a cavity and the antenna is positioned at the substrate edge, or even a monolithic antenna integration.

\section{B. Cylindrical Parabolic Reflector}

The reflector's surface contour can descriptively be derived by means of Fig. 2. The antenna's phase center is positioned in the origin of the coordinate system at the point $O$. The contour of the reflector with the focal length $F_{0}$ is shaped in a way that all reflected waves interfere coherently in direction of the $y$-axis. The point $P(x, y)$ describes the shape of the contour.

The condition that all reflected waves interfere coherently in direction of the $y$-axis is

$$
\overline{O P}+\overline{P Q}=F_{0} .
$$

To derive the shape of the contour as a Cartesian function, the segments $\overline{O P}$ and $\overline{P Q}$ can be expressed as

$$
\overline{O P}=\sqrt{x^{2}+y^{2}}
$$

and as

$$
\overline{P Q}=-y \text {. }
$$

With (2) and (3) following from (1)

$$
\begin{aligned}
\sqrt{x^{2}+y^{2}}-y & =F_{0} \\
x^{2}+y^{2} & =\left(F_{0}+y\right)^{2} \\
x^{2} & =F_{0}^{2}+2 F_{0} y
\end{aligned}
$$

and thus the function for the contour of the cylindrical parabolic reflector as

$$
y=\frac{x^{2}-F_{0}^{2}}{2 F_{0}}
$$

\section{PRototype Design}

To demonstrate the functionality of the concept, a $77-\mathrm{GHz}$ prototype has been designed that consists of a Yagi-Uda antenna on an Alumina substrate, a FR4 substrate, and a reflector.

\section{A. Yagi-Uda Antenna}

Planar Yagi-Uda antennas printed on a high-permittivity substrate were already proposed in 1975 as a way to realize low-cost planar antenna arrays [8]. By using a high-permittivity substrate, the antenna will be extremely compact in terms of freespace wavelengths, and thus an array with a spacing of $\lambda_{0} / 2$ is achievable. Itoh et al. published works on a Quasi-Yagi antenna printed on a high-permittivity substrate fed by a microstrip line [9] and also by a coplanar waveguide (CPW) [10]. Other works on planar Yagi-Uda antennas with simplified feeding structures are [11] and [12]. Neculoiu et al. proposed membrane-supported Yagi-Uda antenna structures on silicon and gallium arsenide semiconductors in the V-and W-band [13], [14]. However, as the semiconductors are generally poor antenna substrates, micromachining technology has to be used to gain sufficient antenna efficiency. This, in fact, enlarges the antenna dimensions since antennas on a thin membrane have to be designed considering the free-space wavelength.

As we designed the antenna for the mere purpose of proving our concept, we adopted the basic design of [10] and [13]. We chose Alumina as antenna substrate because of its properties as a high-permittivity and low-loss substrate and its ability to be processed in thin film technology. The antenna is a planar structure that uses only one metal layer. This enables it to be interconnected to an integrated circuit (IC) via solder balls in a flip-chip attachment. To allow measurement with a coplanar microwave probe, the antenna has been combined with a CPW to coplanar stripline (CPS) balun [15] that could be omitted in a differential circuit. The substrate thickness was chosen to the smallest thickness available $(127 \mu \mathrm{m})$. Yet, this substrate thickness already posed quite a challenge regarding substrate waves. Compared to the works mentioned, the substrate is thicker in terms of wavelengths.

The finally realized Yagi-Uda antenna has three elements, as shown in Fig. 3. The feed element is a folded dipole that is used to get a better matching to the $150 \Omega$ CPS line. The impedance of a single dipole on an Alumina substrate would only be about $40 \Omega$, while the folded dipole has a four-times-larger impedance. The length of the folded dipole is $0.775 \mathrm{~mm}$. At a distance of $0.5 \mathrm{~mm}$ in front of the folded dipole, a director with a length of $0.6 \mathrm{~mm}$ is used. The described balun is directly integrated into the 0.85 - $\mathrm{mm}$-long reflector. We noticed an improved antenna performance using a finite reflector length compared to a truncated ground plane that is used in the works mentioned. All dimensions were optimized to get a suitable pattern and a maximum front-to-back ratio. The distance of the director to the substrate edge was a crucial point in the design, as substrate waves are generated in some cases that cause a radiation to the backside.

\section{B. Reflector}

Extensive simulations have been done to find adequate dimensions for the reflector. The focal length $F_{0}$ has especially been optimized regarding the following criteria:

- a maximum reflector height of $25 \mathrm{~mm}$ to guarantee a small installation depth of the sensor; 


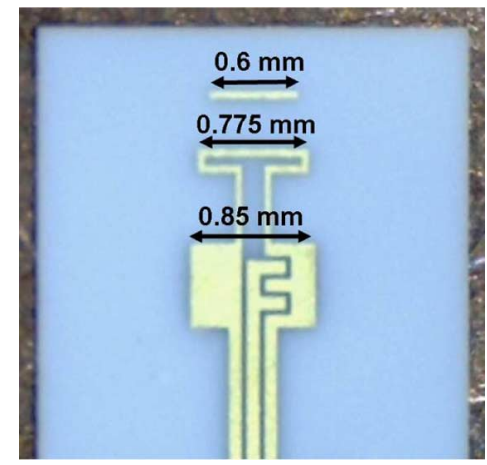

Fig. 3. Yagi-Uda antenna.

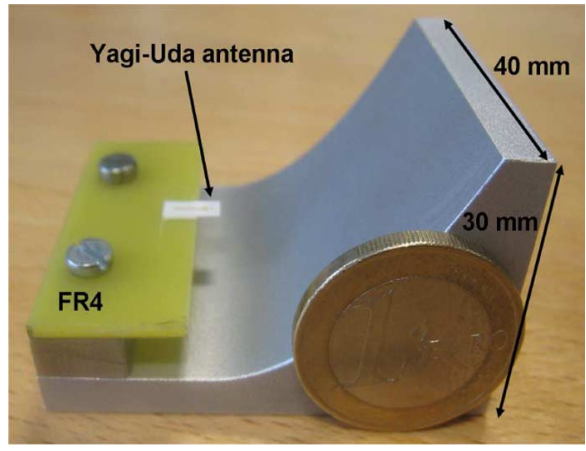

(a) Oblique view

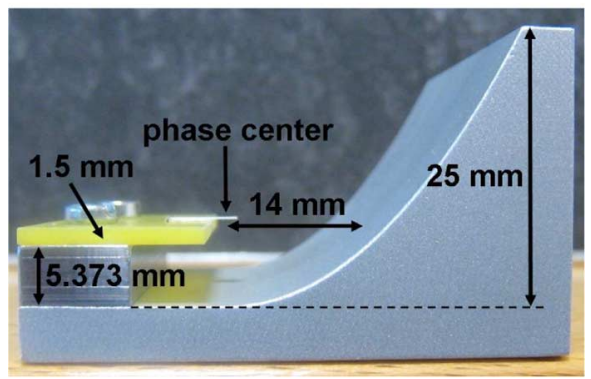

(b) Lateral view

Fig. 4. Manufactured reflector with attached FR4 substrate and antenna.

- a half-power beamwidth (HPBW) in the elevation plane of $10^{\circ}$ at the most;

- a configuration that allows the placement of electric devices on both sides of the PCB

The best result has been achieved with a focal length of $14 \mathrm{~mm}$. A reflector with these dimensions has been manufactured by wire eroding out of an aluminum block, as shown in Fig. 4. On the reflector, a 5.373-mm-thick metal block is used as a spacer on which the 1.5-mm-thick FR4 substrate is attached. The spacing between the FR4 substrate and the reflector could be used for the placement of electric devices in the final sensor. The antenna substrate is attached to the FR4 substrate by an adhesive foil and is positioned in a way that it extends $3 \mathrm{~mm}$ beyond the edge of the FR4 substrate to guarantee ideal radiation surroundings for the antenna.

\section{MEASUREment AND Simulation Results}

The measurements were done with a setup in which a coplanar microwave probe is used to contact the antenna. As

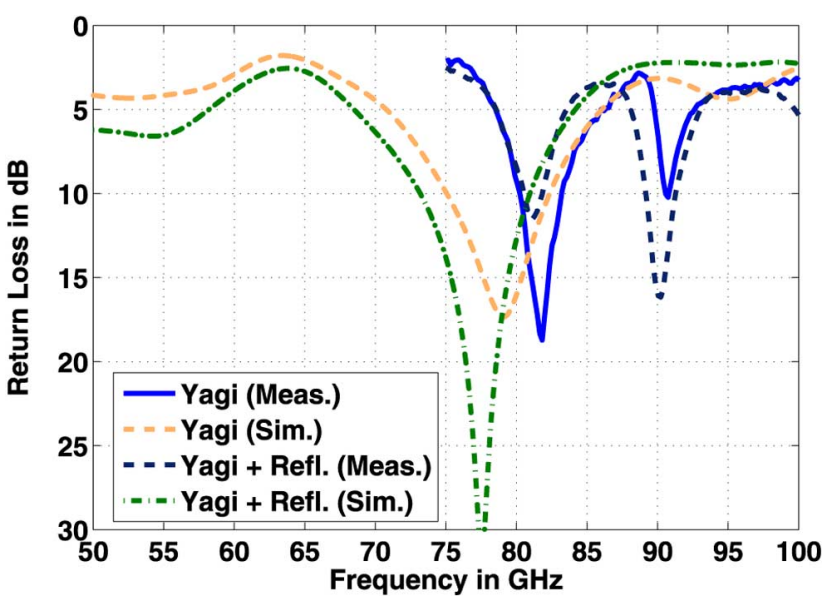

Fig. 5. Measured and simulated input return loss of the Yagi-Uda antenna without and with the reflector.

the probe and the antenna under test (AUT) may not move, a receive antenna is rotated around the AUT in a distance of $60 \mathrm{~cm}$ by two arms and two rotational stages to measure the radiation pattern of the AUT. The setup is described in more detail in [16]. In the setup, the main lobe of the single antenna pointed to the angles $\theta=\psi=90^{\circ}$. Combined with the reflector, the main lobe then pointed to the angles $\theta=\psi=0^{\circ}$ of the setup.

\section{A. Input Return Loss}

The measured and simulated return loss curves of the antenna itself and the antenna combined with the reflector are plotted in Fig. 5. Comparing simulation and measurement of the antenna itself, it can be observed that the resonance shifted to higher frequencies and the bandwidth slightly decreased. This can be explained by the inaccuracy of the substrate permittivity $(9.9 \pm 0.2)$ and the line-width tolerances of the thin film process. A redesign seems necessary here. Additionally, changes can be observed between the return loss of the antenna itself and of the antenna with the reflector. Hence, the reflector should be considered during the final steps of the antenna design.

\section{B. Gain and Radiation Patterns}

1) Yagi-Uda Antenna: The measured and simulated radiation patterns of the Yagi-Uda antenna at a frequency of $80 \mathrm{GHz}$ are plotted in Fig. 6. The plots show the agreement of measured and simulated patterns and gain. Directivity can be observed in both planes, and a measured gain of $5.4 \mathrm{dBi}$ is achieved. The front-to-back ratio could not be measured due to the constricted measurement range. According to simulation results, it is about $11 \mathrm{~dB}$. The $\mathrm{HPBW}$ is $65^{\circ}$ in the E-plane and $54^{\circ}$ in the $\mathrm{H}$-plane. The strongest measured cross-polarization radiation is about $-10 \mathrm{dBi}$ and is assumed to be generated by the balun. The simulation shows a radiation efficiency of $84 \%$.

2) Yagi-Uda Antenna and Cylindrical Parabolic Reflector: Fig. 7 shows the measured and simulated radiation patterns of the Yagi-Uda antenna combined with the cylindrical parabolic reflector at $80 \mathrm{GHz}$. Clearly observable is the focusing in the $\mathrm{H}$-plane through the reflector. A measured gain of $15.8 \mathrm{dBi}$ was achieved, which is more than $10 \mathrm{~dB}$ higher than the gain of the antenna itself. The simulation shows a radiation efficiency of 

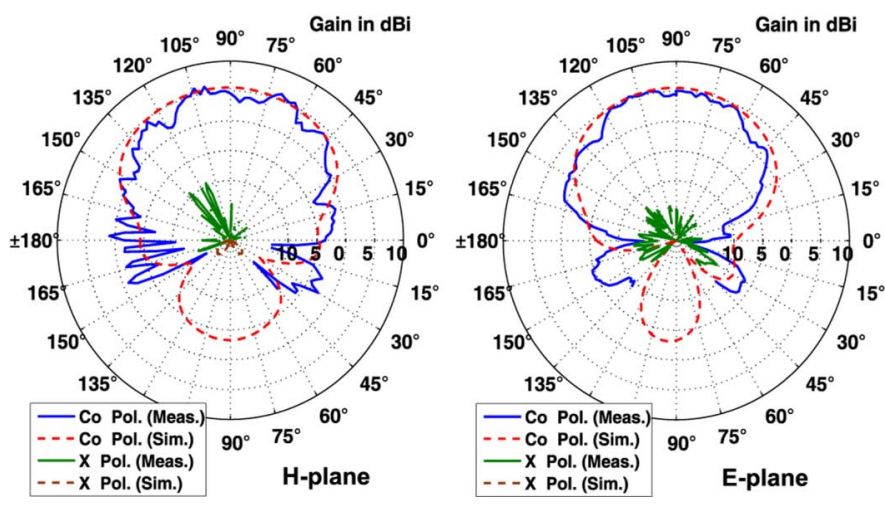

Fig. 6. Gain of the Yagi-Uda antenna.
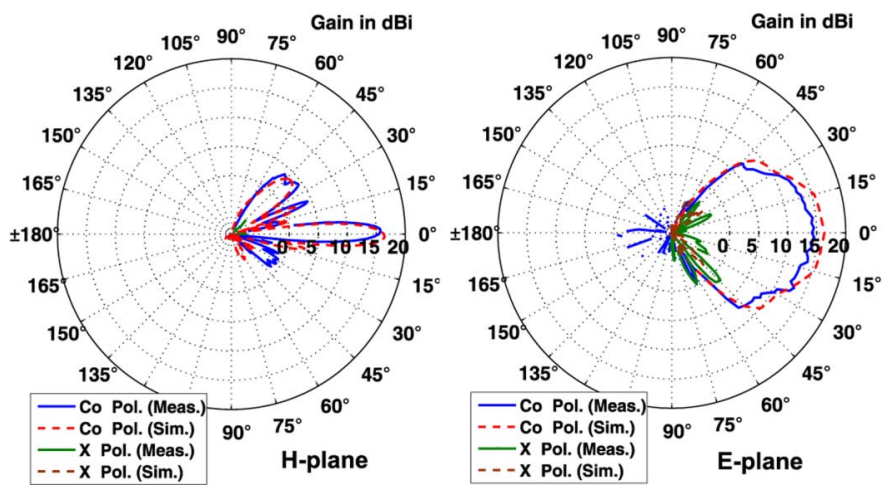

Fig. 7. Gain of the Yagi-Uda antenna with parabolic reflector.

$72 \%$, which means that the efficiency has decreased compared to the antenna itself. The HPBW is $7^{\circ}$ in the H-plane and $42^{\circ}$ in the E-plane. Sidelobes can be observed in the H-plane at about $25^{\circ}$ and $45^{\circ}$. These arise from the fact that the reflector height is limited to $25 \mathrm{~mm}$; hence, some energy is radiated above the reflector, and also some energy is scattered from the reflector edge. To avoid these sidelobes, a slightly higher reflector would have to be used. If, however, the front end is positioned in a way that these sidelobes point to the sky, they should not be critical.

\section{CONCLUSION}

An antenna concept for millimeter-wave radar sensors that desire a fan-beam radiation pattern has been proposed. The proposed assembly reduces the antenna substrate area while maintaining a small installation depth. Due to the fact that the antennas and the active devices can be connected in a flip-chip interconnect, a short and low-loss path for the millimeter-wave signal is guaranteed. As the cylindrical parabolic reflector could easily be integrated into the sensor's metal housing, the concept allows the realization of a cheaper and more compact sensor. The concept has been validated by a 77-GHz prototype with a reflector height of only $25 \mathrm{~mm}$ and a substrate area for the
Yagi-Uda antenna below $2 \mathrm{~mm}^{2}$. The reflector increased the antenna's gain from 5.4 to $15.8 \mathrm{dBi}$ and reduced the HPBW in the $\mathrm{H}$-plane from $54^{\circ}$ to $7^{\circ}$. To attain the desired HPBW in the E-plane and the necessary gain for LRR sensors, planar antenna arrays could be used to realize the different transmit and receive antennas.

\section{ACKNOWLEDGMENT}

The authors would like to thank the CoC Microwave Department of Rohde \& Schwarz GmbH \& Co. KG, Munich, Germany, for supporting their project by manufacturing the Yagi-Uda antenna.

\section{REFERENCES}

[1] M. Schneider, "Automotive radar, status and trends," in Proc. GeMiC, Ulm, Germany, Apr. 2005, pp. 144-147.

[2] R. H. Rasshofer and K. Naab, "77 GHz long range radar systems status, ongoing developments and future challenges," in Proc. EURAD, Oct. 2005, pp. 161-164.

[3] K. M. Strohm, H.-L. Bloecher, R. Schneider, and J. Wenger, "Development of future short range radar technology," in Proc. EURAD, Oct. 2005, pp. $165-168$.

[4] Y. Asano, S. Ohshima, T. Harada, M. Ogawa, and K. Nishikawa, "Proposal of millimeter-wave holographic radar with antenna switching," in Proc. IEEE MTT-S Int. Symp. Dig., 2001, vol. 2, pp. 1111-1114.

[5] A. Kawakubo, S. Tokoro, Y. Yamada, K. Kuroda, and T. Kawasaki, "Electronically-scanning millimeter-wave radar for forward objects detection," SAE World Congr. Trans., vol. 113, pp. 124-131, Mar. 2004.

[6] P. Wenig and R. Weigel, "Analysis of a microstrip patch array fed cylindric lens antenna for $77 \mathrm{GHz}$ automotive radar," in Proc. IEEE AP-S Int. Symp., Jul. 2008, pp. 1-4.

[7] S. K. Reynolds, B. A. Floyd, U. R. Pfeiffer, T. J. Beukema, T. Zwick, J. Grzyb, D. Liu, and B. P. Gaucher, "Progress toward a low-cost millimeter-wave silicon radio," in Proc. IEEE Custom Integr. Circuits Conf., Sep. 2005, pp. 563-570.

[8] W. Wiesbeck, "Dipol- und Yagi-antennen fuer die Mikrowellen-Streifenleitungstechnik," Mikrowellen J., vol. 3, pp. 171-175, Mar. 1975.

[9] W. R. Deal, N. Kaneda, J. Sor, Y. Qian, and T. Itoh, “A new quasi-Yagi antenna for planar active antenna arrays," IEEE Trans. Microw. Theory Tech., vol. 48, no. 6, pp. 910-918, Jun. 2000.

[10] J. Sor, Y. Qian, and T. Itoh, "Coplanar waveguide fed quasi-Yagi antenna," Electron. Lett., vol. 36, no. 1, pp. 1-2, Jan. 2000.

[11] G. Zheng, A. A. Kishk, A. W. Glisson, and A. B. Yakovlev, "Simplified feed for modified printed Yagi antenna," Electron. Lett., vol. 40, no. 8, pp. 464-466, Apr. 2004.

[12] H. K. Kan, R. B. Waterhouse, A. M. Abbosh, and M. E. Bialkowski, "Simple broadband planar CPW-fed quasi-Yagi antenna," IEEE Antennas Wireless Propag. Lett., vol. 6, pp. 18-20, Apr. 2007.

[13] D. Neculoiu, P. Pons, M. Saadaoui, L. Bary, D. Vasilache, K. Grenier, D. Dubuc, A. Muller, and R. Plana, "Membrane supported Yagi-Uda antennae for millimetre-wave applications," Proc. Microw., Antennas Propag., vol. 151, no. 4, pp. 311-314, Aug. 2004.

[14] D. Neculoiu, A. Muller, D. Vasilache, I. Petrini, C. Buiculescu, R. Plana, P. Pons, L. Bary, and M. Saadaoui, "Membrane supported 77 GHz Yagi-Uda antennae: Design and experiment," in Proc. CAS Int. Semiconductor Conf., Oct. 2005, vol. 1, pp. 123-126.

[15] K.-P. Ma, Y. Qian, and T. Itoh, "Analysis and applications of a new CPW-slotline transition," IEEE Trans. Microw. Theory Tech., vol. 47, no. 4, pp. 426-432, Apr. 1999.

[16] S. Beer, G. Adamiuk, and T. Zwick, "Design and probe based measurement of $77 \mathrm{GHz}$ antennas for antenna in package applications," presented at the 39th EuMC, Oct. 2009, to be published. 